\title{
UTILIZAÇÃO DE RMN DE 'H NA DETERMINAÇÃO DA CONFIGURAÇÃO ABSOLUTA DE ÁLCOOIS
}

\author{
Marizeth L. Barreiros e Jorge M. David
}

Instituto de Química, Universidade Federal da Bahia, Campus de Ondina, 40170-290 Salvador - BA

Juceni P. David*

Faculdade de Farmácia, Universidade Federal da Bahia, Campus de Ondina, 40170-290 Salvador - BA

Recebido em 12/7/04; aceito em 17/3/05; publicado na web em 24/8/05

\begin{abstract}
UTILIZATION OF 'H NMR IN THE DETERMINATION OF ABSOLUTE CONFIGURATION OF ALCOHOLS. This review reports the determination of absolute configuration of primary and secondary alcohols by ${ }^{1} \mathrm{H}$ NMR spectroscopy, using the Mosher method. This method consists in the derivatization of an alcohol possessig unknown absolute configuration with one or both enantiomers of an auxiliary reagent. The resulting diastereoisomer spectra are registered and compared, and the chemical shift differences $\left(\Delta \delta^{R, S}\right.$ $=\delta^{R}-\delta^{S}$ ) are measured. The determination of the absolute configuration of the alcohol molecule is based on the correlation between its chiral center and the auxiliary reagent's chiral center. Therefore, the determination of the absolute configuration depends on aromatic ring shielding effects on the substituents of the alcohol as evidenced by the ${ }^{1} \mathrm{H}$ NMR spectrum.
\end{abstract}

Keywords: absolute configuration; alcohols; Mosher's reagent.

\section{INTRODUÇÃO}

Nas últimas décadas houve aumento no desenvolvimento de métodos sensíveis e confiáveis para determinação da configuração absoluta. Esse aumento deveu-se principalmente ao isolamento de grande número de produtos naturais opticamente ativos e aos recentes avanços na área da síntese assimétrica ${ }^{1}$, uma vez que é de interesse das indústrias farmacêuticas e agrícolas a preparação de enantiômeros puros. Neste contexto, a determinação da estereoquímica tem sido de valiosa importância para a química de produtos naturais, assim como para a química sintética tendo em vista que a estrutura de um composto não pode ser considerada determinada até que sua configuração absoluta seja conhecida.

Alguns métodos físicos são utilizados na determinação da estereoquímica absoluta de compostos orgânicos, tais como a cristalografia de Raios-X e a excitação de quiralidade ${ }^{1-3}$. A difração de raios X é o método de maior importância na elucidação estrutural de produtos naturais complexos, sendo o único que permite a determinação da configuração absoluta diretamente. Porém, é limitado pela necessidade da obtenção de monocristais adequados. Entre os métodos de excitação de quiralidade os mais sensíveis são o Dicroísmo Circular (DC) e a Dispersão Ótica Rotatória (DOR), que são utilizados na determinação da configuração absoluta de alguns centros qirais. Estes também apresentam limitações, assim, foram desenvolvidos outros métodos para auxiliar na determinação da configuração absoluta de compostos orgânicos, tais como aqueles realizados através de reações químicas, como o método de Harada e Nakanishi, bem como o método de Horeau, utilizados para determinar configuração absoluta de álcoois ${ }^{1,2,4}$. Estes últimos são baseados na análise do Dicroísmo Circular (DC). Outro método muito utilizado para determinação da configuração absoluta é o método de Mosher. Inicialmente foi introduzido por Raban e Mislow, tendo sido posteriormente adaptado por Mosher, Trost e Dale, entre outros pesquisadores $^{5}$. O método adaptado por Mosher utiliza reagentes de derivatização quiral (RDQ) e posterior análise por RMN de ${ }^{1} \mathrm{H}$. O

*e-mail: juceni@ufba.br interesse despertado por este método baseia-se tanto na possibilidade de trabalhar com amostras em pequenas quantidades, quanto devido a sua simplicidade. Entretanto, este método requer a presença de grupos funcionais específicos na molécula, tais como $-\mathrm{CO}_{2} \mathrm{H}$, $\mathrm{NH}_{2} \mathrm{e}-\mathrm{OH}$, necessários para ligar o substrato ao reagente. Portanto, mostra-se útil apenas para álcoois primários e secundários, aminas primárias e secundárias e para ácidos carboxílicos. Vários reagentes auxiliares têm sido descritos na literatura com essa finalidade ${ }^{2,4,6}$, dentre estes os mais utilizados são ácido $\alpha$-metoxi- $\alpha$-trifluorometil fenil acético MTPA (1), ácido $\alpha$-metoxi-fenil acético MPA (2), ácido 1-naftil- $\alpha$-metoxi acético 1-NMA (3), ácido 2-naftil- $\alpha$-metoxi acético 2-NMA (4), ácido 9-antranil- $\alpha$-metoxi acético 9-AMA (5) e ácido 2-antranil- $\alpha$-metoxi acético 2-AMA (6) (Figura 1).<smiles>CC(F)(F)C(C(=O)O)(c1ccccc1)C(F)(F)F</smiles>

(R)-MTPA 1<smiles>COC(C(=O)O)c1ccc2ccccc2c1</smiles>

(R)-2-NMA 4<smiles>COC(C(=O)O)c1ccccc1</smiles>

(R)-MPA 2<smiles>COc1c2ccccc2cc2ccccc12</smiles>

(R)-9-AMA 5<smiles>CO[C@H](C(=O)O)c1cccc2ccccc12</smiles>

(R)-1-NMA 3

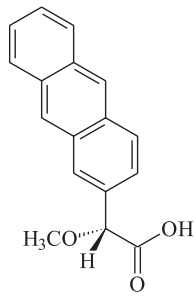

(R)-2-AMA 6
Figura 1. Reagentes de derivatização quiral

\section{O MÉTODO}

O método de Mosher é baseado na dupla derivatização ou na simples derivatização. A dupla derivatização consiste na esterificação do álcool de configuração absoluta desconhecida com os dois 
enantiômeros $(R)$ e $(S)$ do reagente auxiliar quiral, enquanto que a simples derivatização consiste na esterificação do álcool com apenas um dos enantiômeros do reagente auxiliar, $(R)$ ou $(S)$. Nos dois métodos, são registrados os espectros dos dois diasteroisômeros resultantes da reação e comparados, sendo realizada a medida da diferença dos deslocamentos químicos $\Delta \delta^{R, S}$. A determinação da configuração $R / S$ no centro quiral do álcool é feita através da correlação entre esse centro e o centro quiral do reagente auxiliar que é de configuração absoluta conhecida, de acordo com o efeito de proteção e/ou desproteção que o grupo aromático do reagente auxiliar produzirá nos hidrogênios dos substituintes $\mathrm{L}_{1}$ e $\mathrm{L}_{2}$ do álcool.

$\mathrm{O}$ reagente auxiliar deve possuir algumas características estruturais especiais, tais como a presença de um grupo $(\mathrm{Z}=$ grupos carboxilatos, hidroxílicos ou cloretos) capaz de ligar o álcool ao reagente através desse grupo e também de um grupo ( $\mathrm{Y}=$ anel aromático ou grupo insaturado) com forte efeito anisotrópico para produzir efeito de proteção ou desproteção nos substituintes $\mathrm{L}_{1} / \mathrm{L}_{2}$ do álcool. Finalmente, deve possuir um grupo polar $\left(\mathrm{R}_{1}\right.$ e $\left.\mathrm{R}_{2}\right)$ necessário para auxiliar a fixação da molécula na conformação preferida ${ }^{7}$.

Alguns critérios devem ser levados em consideração para que o método possa ser aplicado. Entre estes, o espectro de RMN de ${ }^{1} \mathrm{H}$ deve apresentar, claramente, sinais distinguíveis e identificáveis de hidrogênio nos dois substituintes $\mathrm{L}_{1} / \mathrm{L}_{2}$ do álcool. Deste modo, normalmente utiliza-se equipamentos de campo maior (> 7,05 Tesla). Outra característica requerida estabelece que o álcool deve apresentar hidrogênio nos dois substituintes $\mathrm{L}_{1} / \mathrm{L}_{2}$, e que os sinais de deslocamento químicos $(\Delta \delta)$ nos substituintes $\mathrm{L}_{1} / \mathrm{L}_{2}$ sejam opostos. Deste modo, espera-se que todos os deslocamentos dos hidrogênios de $\mathrm{L}_{1}$ apresentem mesmo sinal de $\Delta \delta$, e todos os deslocamentos dos hidrogênios de $\mathrm{L}_{2}$ também apresentem mesmo sinal de $\Delta \delta$.

\section{REAGENTES}

\section{$O$ reagente MTPA}

O reagente de Mosher MTPA (1) foi introduzido por Mosher ${ }^{8}$ em 1969. Este é encontrado comercialmente como ácido ou cloreto de ácido em ambas as formas enantiomericamente puras. É um dos reagentes auxiliares mais populares para reconhecimento da estereoquímica absoluta de álcoois secundários e aminas primárias, ambos $\alpha$-substituídos ${ }^{7,9}$.

Os ésteres de MTPA, de acordo com cálculos semiempíricos, são constituídos de três confôrmeros principais ${ }^{10,11}$ de baixa energia em equilíbrio e com populações similares dos confôrmeros $s \boldsymbol{p}_{1}$, $s p_{2}$ e $\boldsymbol{a} \boldsymbol{p}_{1}$ independentemente da estrutura do álcool que são gerados pelas rotações em torno das ligações $\mathrm{C} \alpha-\mathrm{C}=\mathrm{O}$ e $\mathrm{C} \alpha-\mathrm{Ph}$.

Conforme pode ser observado na Figura 2, em alguns confôrmeros o anel aromático produz proteção e em outros, desproteção. Assim, nos ésteres de $(R)$-MTPA o grupo $\mathbf{L}_{\mathbf{1}}$ está localizado na região de desproteção do anel aromático no confôrmero $a p_{1}$, e não é afetado nos confôrmeros $s p_{1}$ e $s p_{2}$. Entretanto, o grupo em $\mathbf{L}_{2}$ está localizado na região de proteção do anel aromático no confôrmero $s p_{1}$ e na região de desproteção do confôrmero $s p_{2}$, não sendo afetado no confôrmero $a p_{1}$ (Figura 2a). Nos ésteres de $(S)$ MTPA o substituinte $\mathbf{L}_{1}$ está localizado na área de proteção do anel aromático no confôrmero $s p_{1}$ e na região de desproteção no confôrmero $s \boldsymbol{p}_{2}$, não sendo afetado no confôrmero $\boldsymbol{a} \boldsymbol{p}_{1}$. Enquanto que, nestes ésteres, o substituinte $\mathbf{L}_{2}$ está na região de desproteção do anel aromático no confôrmero $a p_{1}$, não sendo afetado nos confôrmeros $\boldsymbol{s} \boldsymbol{p}_{1}$ e $\boldsymbol{s} \boldsymbol{p}_{2}$, respectivamente (Figura 2b). Neste caso, os efeitos anisotrópicos de proteção e desproteção do anel aromático nos confôrmeros $\boldsymbol{s p}_{1}$ e $\boldsymbol{s} \boldsymbol{p}_{2}$ são cancelados mutuamente. Portanto, o efeito mais acentuado no espectro de $\mathrm{RMN}$ de ${ }^{1} \mathrm{H}$ será aquele do confôrmero $a p_{l}$, que é de desproteção. Os valores de $\Delta \delta^{R, S}$ são calculados de acordo com as fórmulas: $\Delta \delta^{R, S} \mathrm{~L}_{1}=\left(\delta^{R} \mathrm{~L}_{1}-\delta^{S} \mathrm{~L}_{1}\right) \mathrm{e}$ $\Delta \delta^{R, S} \mathrm{~L}_{2}=\left(\delta^{R} \mathrm{~L}_{2}-\delta^{S} \mathrm{~L}_{2}\right)$. Tendo em vista que nos ésteres de MTPA o confôrmero $a p_{1}$ predomina, o substituinte $\mathbf{L}_{1}$ é mais desprotegido nos ésteres do isômero $(R)$-MTPA, significando que $\left(\Delta \delta^{R, S} \mathrm{~L}_{1}>0\right)$, enquanto que o substituinte $\mathbf{L}_{2}$ é mais desprotegido nos ésteres do isômero $(S)$-MTPA que no isômero $(R)$-MTPA, de modo que $\Delta \delta^{R, S} \mathrm{~L}_{2}<0$.

a)

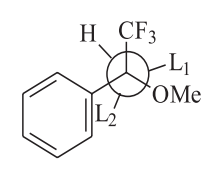

$\mathrm{sp}_{1}$

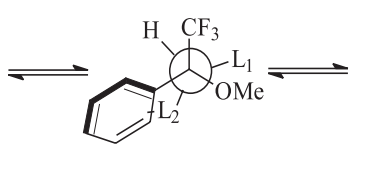

$\mathrm{sp}_{2}$

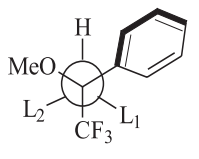

$\mathrm{ap}_{1}$ proteção em $\mathrm{L}_{2}$ desproteção em $\mathrm{L}_{2}$ desproteção em $\mathrm{L}_{1}$

b)

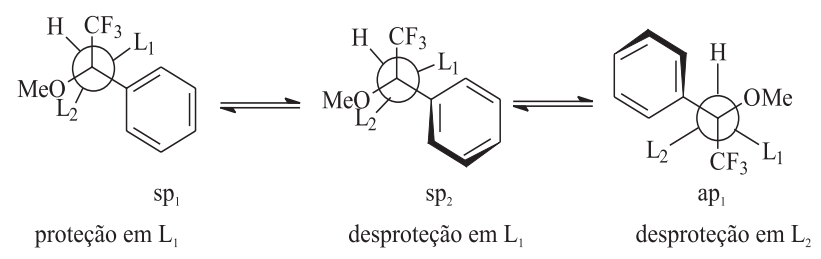

Figura 2. Principais confôrmeros dos ésteres de MTPA (1)

\section{$O$ reagente MPA}

O reagente MPA (2) foi introduzido por Raban e Mislow ${ }^{12}$ e, assim como o reagente de Mosher, é comercialmente encontrado na forma de ácido ou cloreto de ácido nas duas configurações enantiomericamente puras, sendo também um dos reagentes mais utilizados para determinação da configuração absoluta de álcoois secundários- $\alpha$-substituídos $2,7,10,12,13$.

Diferentemente dos ésteres de MTPA, os ésteres de MPA de acordo com cálculos semiempíricos ${ }^{2,10,13}$ são constituídos de 2 confôrmeros principais de baixa energia independentemente da estrutura do álcool: $\boldsymbol{s} \boldsymbol{p}$ e $\boldsymbol{a} \boldsymbol{p}$. Estes são gerados pelas rotações em torno das ligações $\mathrm{C} \alpha-\mathrm{C}=\mathrm{O}$ e $\mathrm{C} \alpha-\mathrm{Ph}$, sendo o confôrmero $\boldsymbol{s p}$ mais estável (Figura 3).

De acordo com estes resultados nos ésteres de $(R)$-MPA, o grupo $\mathbf{L}_{1}$ está na região de proteção do anel aromático no confôrmero $s p$ e não é afetado no confôrmero ap, enquanto que o grupo $\mathbf{L}_{2}$ é protegido no confôrmero $a p$ e não está sendo afetado no confôrmero $\boldsymbol{s p}$ (Figura 3a). Por outro lado, nos ésteres de (S)-MPA o grupo $\mathbf{L}_{2}$ está na região de proteção do anel aromático no confôrmero $\boldsymbol{s p}$ e não é afetado no confórmero $a p$, enquanto que o grupo $\mathbf{L}_{1}$ está na zona de proteção do anel aromático no confôrmero ap e não está sendo afetado no confôrmero $\boldsymbol{s p}$ (Figura 3b). Alternativamente, nos ésteres de MPA o confôrmero predominante é $\boldsymbol{s} \boldsymbol{p}$. Deste modo, o grupo $\mathbf{L}_{1}$ é mais protegido nos ésteres de $(R)$-MPA que nos ésteres do enantiômero $(S)$-MPA. Assim $\Delta \delta^{R, S} \mathrm{~L}_{1}<0$, enquanto que o grupo $\mathbf{L}_{2}$ está mais protegido nos ésteres do enantiômero $(S)$-MPA, de modo que $\Delta \delta^{R, S} \mathrm{~L}_{2}>0$.

\section{Comparação entre os reagentes MTPA e MPA}

Pode-se observar que entre os ésteres de MTPA (1) e MPA (2), os ésteres derivados de MTPA apresentam comportamento 
a)

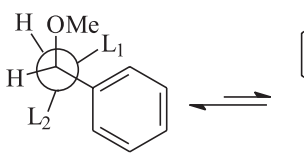

sp

proteção em $\mathrm{L}_{1}$

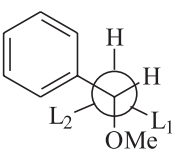

ap b)

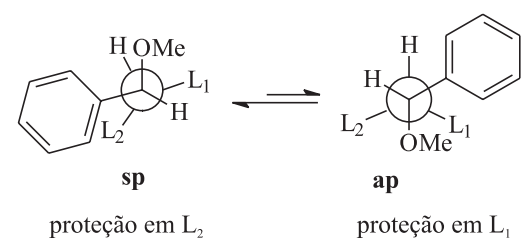

Figura 3. Principais confôrmeros dos ésteres de MPA (2)

conformacional mais complexo em torno da ligação $\mathrm{C} \alpha-\mathrm{Ph}$. Nesses ésteres derivados de MTPA, o anel aromático apresenta três orientações e não exerce forte preferência conformacional por nenhuma delas. Observa-se leve predominância do confôrmero $a p_{1}$, que exerce efeito de desproteção, tanto sobre o grupo $\mathbf{L}_{1}$ quanto $\mathbf{L}_{2}$, devido ao cancelamento mútuo dos outros dois confôrmeros $s p_{1}$ e $\boldsymbol{s} \boldsymbol{p}_{2}$, enquanto que nos ésteres de MPA o anel aromático tem preferência conformacional, sendo o confôrmero $s p$ o principal. Esse exerce efeito de proteção, de forma que se observam maiores valores de deslocamentos químicos $\Delta \delta^{R, S}$. Isso possibilita que o MPA seja melhor escolha de reagente para a determinação da configuração absoluta de álcoois secundários que o MTPA.

\section{Os reagentes AMAA}

Os derivados aril metoxilados do ácido acético (AMAA) 1NMA (3), 2-NMA (4), 9-AMA (5) e 2-AMA (6) são utilizados para determinação da configuração absoluta de álcoois secundários- $\alpha$-substituídos ${ }^{2,14}$ e álcoois primários- $\beta$-substituídos ${ }^{15}$. Estas substâncias foram desenvolvidas para melhorar os valores da variação dos deslocamentos químicos $\Delta \delta^{R, S}$ obtidos com MPA e MTPA. Este efeito pode ser obtido através da substituição do grupo fenila do MPA por outro grupo com sistema aromático mais eficiente, como naftila e antranila, capaz de produzir deslocamentos químicos cerca de 3 vezes maiores que aqueles obtidos com MPA 2,14 $^{2}$ (Tabela 1).

A eficiência destes grupos é principalmente devida ao aumento de tamanho do anel aromático que, conseqüentemente, aumenta a área do cone de proteção do sistema aromático e, por outro lado, devido a fatores estéricos, onde a conformação mais estável é favorecida. Assim, observa-se o aumento na população relativa deste confôrmero produzindo aumento nos valores de $\Delta \delta^{R, S}$. Os reagentes 1-NMA (3) e 9-AMA (5) são utilizados para determinação da configuração absoluta principalmente de álcoois cíclicos, enquanto que os reagentes 2-NMA (4) e 2-AMA (6) são empregados para álcoois lineares de cadeia longa ${ }^{14}$.

Dentre estes reagentes, o 9-AMA (5) e o 2-AMA (6) são os mais eficientes para determinação da configuração absoluta de álcoois primários ${ }^{15}$, pois apresentam área do cone magnético maior, tendo consequientemente, uma área de maior alcance. Nos álcoois primários, a distância entre o centro assimétrico do álcool e o anel aromático do reagente auxiliar é maior que em álcoois secundários. Deste modo, os valores de $\Delta \delta^{R, S}$ em álcoois primários são maiores e mais confiáveis com o 9-AMA (5), que com aqueles obtidos com os outros reagentes que têm um grupo fenila. Estes reagentes apresentam comportamento conformacional igual ao MPA (2) apresentando 2 confôrmeros principais $\boldsymbol{s} \boldsymbol{p}$ e $\boldsymbol{a} \boldsymbol{p}$, onde o confôrmero $\boldsymbol{s} \boldsymbol{p}$ é o mais estável.

Este método pode ser aplicado tanto para álcoois secundários- $\alpha$ substituídos quanto para álcoois primários- $\beta$-substituídos, com grande variedade de grupos e substituições como os exemplos do Quadro 1. Porém, este não é confiável para álcoois primários altamente impedidos, tais como os exemplificados em 8-10. Isto devido às dificuldades destes adotarem a conformação ideal, levando a valores de $\Delta \delta^{R, S}$ muito baixos. Ele também não pode ser aplicado para álcoois primários- $\gamma$-substituídos, ou seja, com o centro quiral distante de duas ligações, tais como em 11 e 12. A partir destes dois exemplos pode-se observar uma distribuição irregular nos sinais. Este fato pode ser atribuído, principalmente, tanto a fatores estéricos quanto à distância entre o centro assimétrico do álcool e o anel aromático do reagente auxiliar, haja vista que o efeito de proteção do anel aromático diminui com a distância. No exemplo 11 todos os valores de $\Delta \delta^{R, S}$ para os substituintes $\mathrm{L}_{1} / \mathrm{L}_{2}$ são positivos e, no exemplo $12 \mathrm{em}$ um dos substituintes verifica-se valor positivo e negativo de $\Delta \delta^{R, S}$ do mesmo lado, invalidando o método.

Outra maneira de aumentar os valores da variação dos deslocamentos químicos $\Delta \delta^{R, S}$ daqueles obtidos com MPA é através da diminuição da temperatura no probe do RMN.

O primeiro passo a ser seguido é a modificação da diferença de energia entre os dois confôrmeros, através da variação da temperatura. De acordo com a equação de Boltzman, o decréscimo de $100^{\circ}$ C no caso dos ésteres de MPA leva ao aumento em cerca de $10 \%$ na população relativa do confôrmero $\boldsymbol{s p}$, o que corresponde ao aumento de, no mínimo, $33 \%$ no valor ${ }^{2,15}$ de $\Delta \delta^{R, S}$.

Estudos realizados sobre a variação de $\Delta \delta^{R, S}$ com a temperatura para os ésteres de MPA (2) e para os ésteres dos outros AMAA (2NMA (4) e 9-AMA (5) mostraram que os valores de $\Delta \delta^{R, S}$ destes últimos reagentes são similares àqueles obtidos com os ésteres de MPA (2). Isto provavelmente ocorre devido a fatores estéricos que impedem a desestabilização do equilíbrio, fazendo com que os va-

Tabela 1. Valores de $\Delta \delta$ para os ésteres dos AMAA.: 9-AMA(5), 1-NMA(3), 2-NMA(4), MTPA(1).

\begin{tabular}{|c|c|c|c|c|c|c|}
\hline \multicolumn{7}{|c|}{$\Delta \delta \mathrm{R}, \mathrm{s}$} \\
\hline Reagente & álcool & $\mathbf{C H}_{3}(1)$ & $\mathbf{C H}_{2}(2)$ & $\mathrm{CH}_{2}$ (3) & $\mathbf{C H}_{2}$ (4) & СН(5) \\
\hline MTPA & & 0,07 & 0,11 & 0,04 & $-0,07$ & $-0,13$ \\
\hline 1-NMA & & 0,23 & 0,31 & 0,13 & $-0,15$ & $-0,27$ \\
\hline 2-NMA & 13 (Quadro 1) & 0,37 & 0,55 & 0,22 & $-0,23$ & $-0,47$ \\
\hline 9-AMA & & 0,59 & 0,90 & 0,35 & $-0,36$ & $-0,37$ \\
\hline & & $\mathbf{C H}_{3}(8)$ & $\mathrm{CH}_{3}$ (9) & $\mathbf{C H}_{3}(10)$ & & \\
\hline MTPA & & $-0,17$ & $-0,04$ & 0,07 & & \\
\hline 1-NMA & 6 (Quadro1) & $-0,52$ & $-0,065$ & 0,11 & & \\
\hline 2-NMA & & $-0,36$ & $-0,35$ & 0,04 & & \\
\hline 9-AMA & & $-0,79$ & $-0,75$ & 0,19 & & \\
\hline
\end{tabular}


<smiles>CCC(C)CO</smiles><smiles>CC1(C)C2CCC(CO)C1C2</smiles><smiles>CC1(C)OCC(CO)O1</smiles><smiles>COC(=O)C(C)CO</smiles><smiles>CC(CO)CBr</smiles><smiles>CC(C)C1CCC(Br)CC1O</smiles><smiles>CC(O)C(C)(C)C</smiles><smiles>CC1(C)CCC[C@]2(C)CCCC1C2(C)O</smiles><smiles>CC(C)[C@H]1OC1CO</smiles><smiles>C=CC1CCC(C)C2(CC)CCC(CC)C1C2</smiles>
8

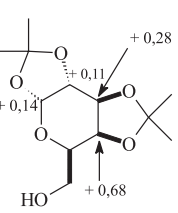

9<smiles>C=C(C=CC(C)CCC(C)CCC(=O)c1ccc(OC)cc1C)C(C)CC</smiles>

13
Quadro 1. Exemplos de álcoois secundários e álcoois primários. Reproduzido das refs. 14 e 15, com permissão da Elsevier

lores de $\Delta \delta^{R, S}$ não cresçam na mesma proporção que quando é usado o MPA (2) (Figura 4). Melhores resultados são observados somente com o MPA (2). Desta forma, com o aumento da população do confôrmero $\boldsymbol{s} \boldsymbol{p}$ o substituinte $\mathbf{L}_{\mathbf{1}}$ é deslocado no éster do enantiômero $(R)$-MPA para campo de proteção, enquanto o substituinte $\mathbf{L}_{2}$ é deslocado para campo de desproteção. Assim $\Delta \delta^{\mathrm{T} 1 \mathrm{~T} 2}$ $\mathrm{L}_{1}>0$ e $\Delta \delta^{\mathrm{T} 1, \mathrm{~T} 2} \mathrm{~L}_{2}<0$, respectivamente. Ao contrário, com o éster do enantiômero $(S)$-MPA o grupo $\mathbf{L}_{\mathbf{1}}$ é deslocado para campo de desproteção e o grupo $\mathbf{L}_{2}$ é deslocado para campo de proteção. Sendo assim, $\Delta \delta^{\mathrm{T} 1, \mathrm{~T} 2} \mathrm{~L}_{1}<0$ e $\Delta \delta^{\mathrm{T} 1 \mathrm{~T} 2} \mathrm{~L}_{2}>0$, respectivamente.

\section{Condições experimentais para registro dos espectros dos derivados}

\section{Dupla derivatização}

As condições operacionais de RMN devem ser à temperatura ambiente e usar como solvente, $\mathrm{CDCl}_{3}$ Os espectros de $\mathrm{RMN}$ dos dois enantiômeros devem ser registrados na mesma temperatura, concentração e solvente. Além disso, é importante utilizar a mesma referência interna para ajustar os valores de deslocamentos.

A determinação da configuração absoluta através da dupla derivatização requer ambos os enantiômeros, e o assinalamento da configuração $R / S$ deve-se seguir as etapas como determinadas na Figura 5: esterificação do álcool com os enantiômeros $(R)$ e o $(S)$ do reagente auxiliar, registro dos espectros de $\mathrm{RMN}$ de ${ }^{1} \mathrm{H}$ dos dois diasteroisômeros; em seguida, deve-se escolher arbitrariamente os substituintes $\mathrm{L}_{1}$ e $\mathrm{L}_{2}$ e atribuir corretamente os valores de RMN de ${ }^{1} \mathrm{H}$ destes grupos $\mathrm{L}_{1}$ e $\mathrm{L}_{2}$ nos dois diasterômeros $(R)$ e $(S)$. Assim, comparam-se os valores de deslocamento químicos destes substituintes $\mathrm{L}_{1} \mathrm{e}$ $\mathrm{L}_{2}$ em ambos os derivados e calculam-se as diferenças de $\Delta \delta^{R, S}$. Os parâmetros de cálculo da diferença de $\Delta \delta^{R, S}$ são definidos como os valores de deslocamento químico para o hidrogênio do substituinte $\mathrm{L}_{1}$ no derivado auxiliar- $R$ menos o deslocamento químico do mesmo hi-
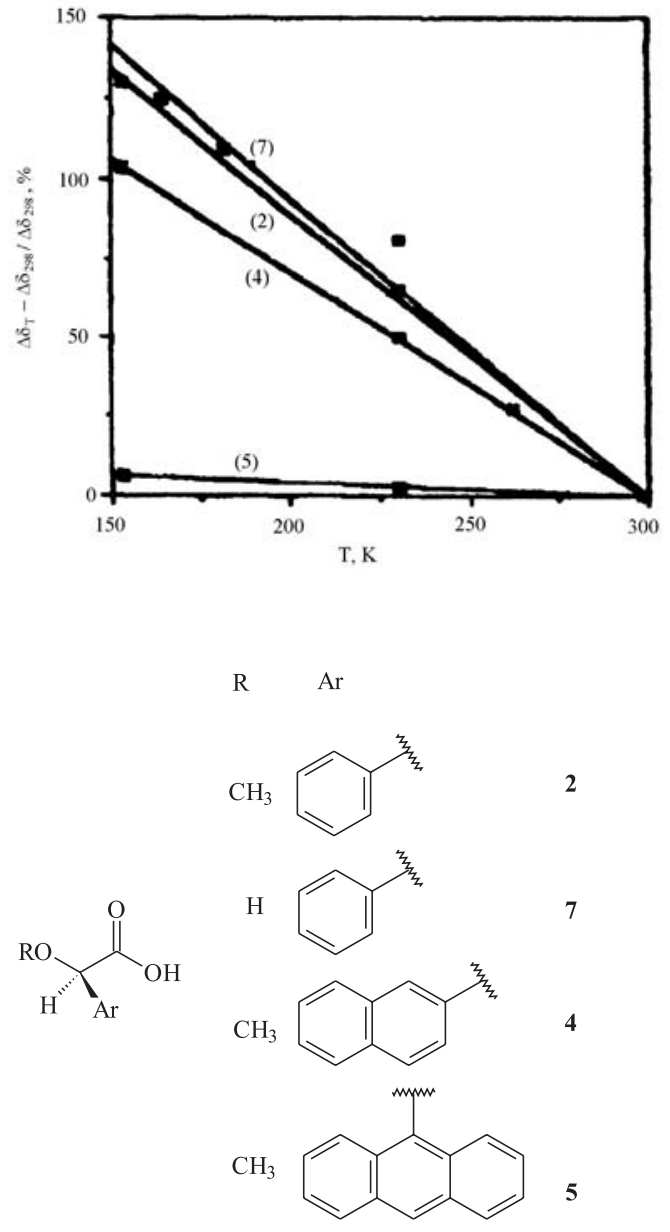

Figura 4. Gráfico do aumento proporcional para os valores de $\Delta \delta^{R, S}$ para o grupo metila dos AMAA, 2 (MPA), 4 (2-NMA), 5 (9-AMA) e 7 com o decréscimo da temperatura no probe. Reproduzido da ref. 15, com permissão da Elsevier

drogênio do grupo $\mathrm{L}_{1}$ do derivado auxiliar- $S$, tal como nas equações $\Delta \delta^{R, S} \mathrm{~L}_{1}=\delta^{R} \mathrm{~L}_{1}-\delta^{S} \mathrm{~L}_{1}$ e $\Delta \delta^{R, S} \mathrm{~L}_{2}=\delta^{R} \mathrm{~L}_{2}-\delta^{S} \mathrm{~L}_{2}$. Assim, tem-se como resultado a determinação da configuração $\mathrm{R} / \mathrm{S}$ baseada na correlação entre a estereoquímica no centro quiral do reagente auxiliar e os deslocamentos químicos dos substituintes $\mathrm{L}_{1}$ e $\mathrm{L}_{2}$ nos dois diasteroisômeros resultantes da reação. Deste modo, o assinalamento da configuração depende do efeito de proteção que o grupo aromático do reagente auxiliar produz nos hidrogênios dos substituintes $\mathrm{L}_{1}$ e $\mathrm{L}_{2}$ do álcool. Se os valores de $\delta$ do substituinte $\mathrm{L}_{1}$ ou do substituinte $\mathrm{L}_{2}$ são deslocados para campo de proteção significa que este grupo está localizado no mesmo lado do plano do anel aromático.

\section{Simples derivatização}

As condições de RMN devem contemplar baixa temperatura $\left(-70{ }^{\circ} \mathrm{C}\right)$ e uso do solvente $\mathrm{CS}_{2} / \mathrm{CD}_{2} \mathrm{Cl}_{2}$ (4:1). A determinação da configuração absoluta requer o uso de apenas um dos enantiômeros. Desse modo, o assinalamento da configuração $\mathrm{R}$ ou $\mathrm{S}$ é realizado a partir da esterificação do álcool com um dos enantiômeros $(R)$ ou $(S)$ do reagente auxiliar MPA (2) ${ }^{16}$ (Figura 6) e, registro do espectro de RMN de ${ }^{1} \mathrm{H}$ do diasteroisômero a temperatura ambiente $\mathbf{T}_{1}$ e do espectro à baixa temperatura $\mathbf{T}_{2}$. O parâmetro para cálculo da diferença de $\Delta \delta^{\mathrm{Tl}, \mathrm{T} 2}$ é definido como o valor de deslocamento químico para o hidrogênio do substituinte $\mathrm{L}_{1}$ no derivado à temperatura ambiente $\mathbf{T}_{1}$ subtraindo-se o valor do deslocamento químico do mesmo hidrogênio deste derivado a baixas temperaturas $\mathbf{T}_{2}$, tal como dado na equação $\Delta \delta^{\mathrm{Tl}, \mathrm{T2}} \mathrm{L}_{1}=\delta^{\mathrm{T1}} \mathrm{L}_{1}$ 


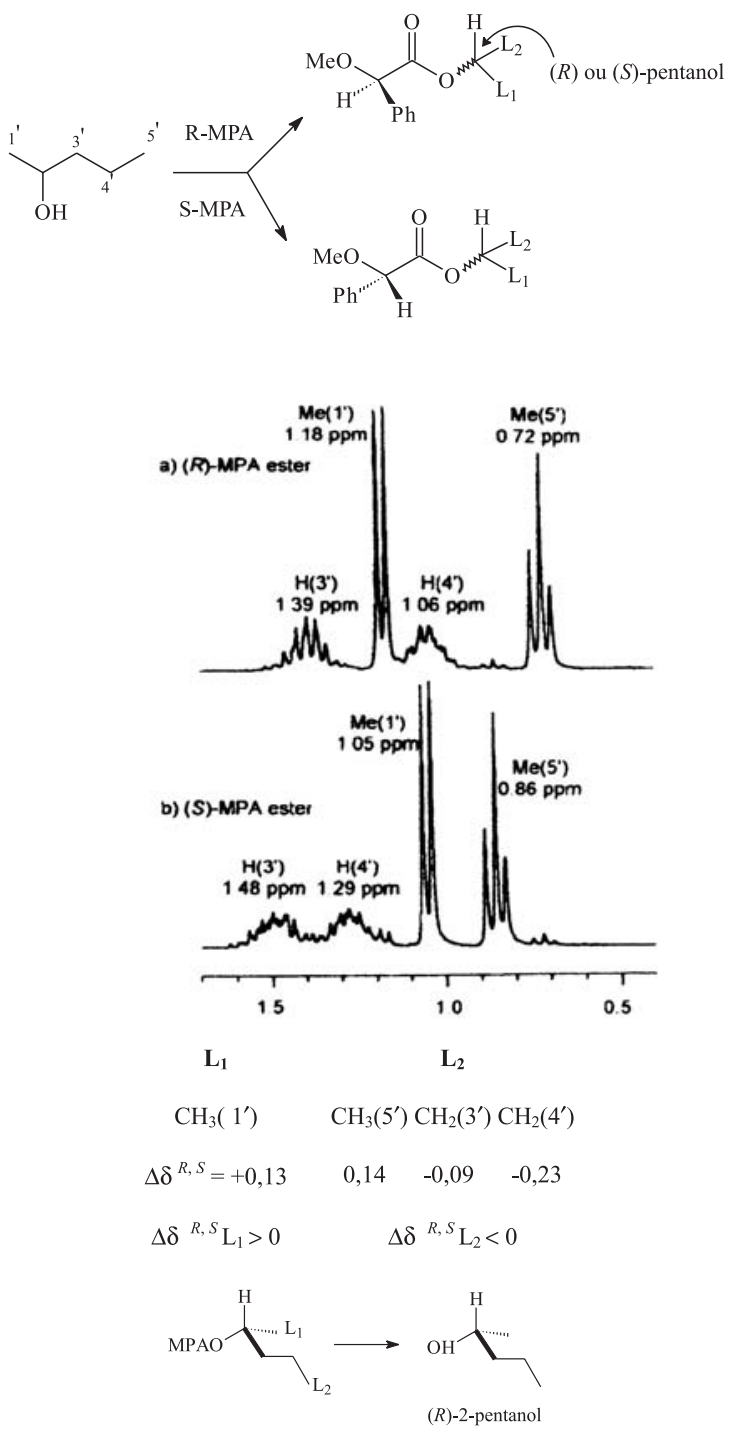

Figura 5. Procedimentos para determinação da configuração do (R)-2pentanol. Reproduzido da ref. 6, com permissão da Elsevier

- $\delta^{\mathrm{T} 2} \mathrm{~L}_{1}$ e $\Delta \delta^{\mathrm{T} 1 \mathrm{~T} 2} \mathrm{~L}_{2}=\delta^{\mathrm{T} 1} \mathrm{~L}_{2}-\delta^{\mathrm{T} 2} \mathrm{~L}_{2}$. O assinalamento da configuração R/ $\mathrm{S}$ é idêntico ao de álcoois na dupla derivatização.

\section{CONCLUSÕES}

A técnica de RMN apresenta vantagens em relação a outras técnicas para determinação da configuração devido a sua simplicidade, além da possibilidade de trabalhar com pequenas quantidades de amostra.

O método de Mosher é confiável para determinação da configuração absoluta de álcoois primários e secundários, tanto cíclicos como acíclicos, desde que obedeçam os parâmetros conforme indicados no Método para que a técnica possa ser aplicada. Porém, não é confiável para determinação da configuração absoluta de moléculas de estruturas muito complexas e com vários grupos hidroxílicos.

O melhor reagente auxiliar para determinação da configuração absoluta de álcoois secundários- $\alpha$-substituídos é o MPA (2) e para álcoois primários- $\beta$-substituídos é o 9-AMA.

\section{REFERÊNCIAS}

1. Rinaldi, P. L.; Prog. Nucl. Magn. Reson. Spectrosc. 1982, 15, 291.
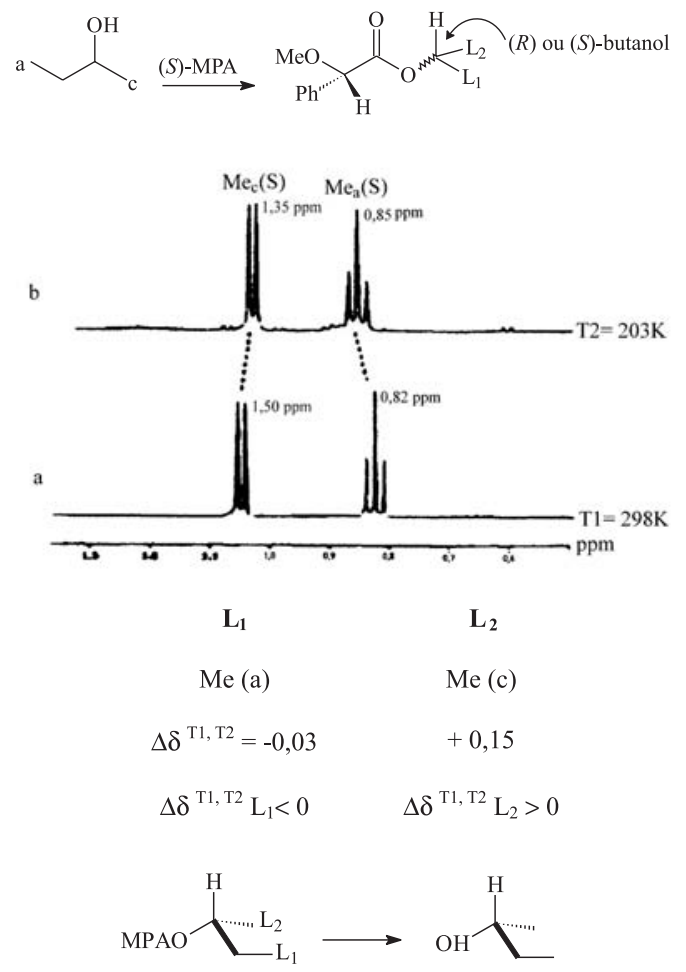

(R)-2-butanol

Figura 6. Procedimentos para assinalamento da configuração do (R)-2butanol. Reproduzido da ref. 5, com permissão da Elsevier

2. Seco, J. M.; Latypov, S. K.; Quiñoá, E.; Riguera, R.; Tetrahedron 1997, $53,8541$.

3. Ohtani, I.; Kusumi, T.; Kashman, Y.; Kakisawa, H.; J. Am. Chem. Soc. 1991, 113,4092

4. Eliel, E. L.; Wilen, S. H.; Mander, L. N.; Stereochemistry of Organic Compounds, New York, 1994, p. 140.

5. Latypov, S. K.; Ferreiro, M. J.; Quiñoá, E.; Riguera, R.; J. Am. Chem. Soc. 1998, 120, 877; Seco, J. M.; Latypov, S. K.; Quiñoá, E.; Riguera, R.; Tetrahedron: Asymmetry 1995, 6, 107.

6. Seco, J. M.; Quiñoá, E.; Riguera, R.; Tetrahedron: Asymmetry 2001, 12, 2915; Fukushi, Y.; Yajima, C.; Mizutani, J.; Tetrahedron Lett. 1994, 35, 599.

7. Seco, J. M.; Quiñoá, E.; Riguera, R.; J. Org. Chem. 1999, 64, 4669.

8. Parker, D.; Chem. Rev. 1991, 91, 1441.

9. Chataigner, I.; Lebreton, J.; Durand, D.; Guingant, A.; Villiéras, J.; Tetrahedron Lett. 1998, 39, 1759; Kusumi, T.; Fujita, Y.; Ohtani, I.; Kakisawa, H.; Tetrahedron Lett. 1991, 32, 2923; Velten, R.; Steglich, W.; Anke, T.; Tetrahedron: Asymmetry 1994, 5, 1229; Kouda, K.; Ooi T.; Kusumi, T.; Tetrahedron Lett. 1999, 40, 3005; Ohtani, I.; Kusumi, T.; Kashman, Y.; Kakisawa, H.; J. Org. Chem. 1991, 56, 1296.

10. Latypov, S. K.; Seco, J. M.; Quiñoá, E.; Riguera, R.; J. Org. Chem. 1996 , $61,8569$.

11. Harada, K.; Shimizu, Y.; Kawakami, A.; Kiyonaga, F.; Tetrahedron Lett. 1999, 40, 9081.

12. Trost, B. M.; Belletire, J. L.; Godleski, S.; McDougal, P. G.; Balkovec, J. M.; J. Org. Chem. 1986, 51, 2370.

13. Latypov, S. K.; Seco, J. M.; Quiñoá, E.; Riguera, R.; J. Org. Chem. 1995, 60, 504.

14. Kouda, K.; Kusumi, T.; Ping, X.; Kan, Y.; Hashimoto, T.; Asakawa, Y.; Tetrahedron Lett. 1996, 37, 4541; Seco, J. M.; Latypov, S. K.; Quiñoá, E.; Riguera, R.; Tetrahedron Lett. 1994, 35, 2921; Kusumi, T.; Takahashi, H.; Xu, P.; Fukushima, T.; Asakawa, Y.; Hashimoto, T.; Kan, Y.; Inouye, Y.; Tetrahedron Lett. 1994, 35, 4397; Takahashi, H.; Kusumi, T.; Tetrahedron Lett. 1996, 36, 7087; Takahashi, H.; Iwashima, M.; Iguchi, K.; Tetrahedron Lett. 1999, 40, 333.

15. Latypov, S. K.; Ferreiro, M. J.; Quiñoá, E.; Riguera, R.; J. Am. Chem. Soc. 1998, 120, 4741; Ferreiro, M. J.; Latypov, S. K.; Quiñoá, E.; Riguera, R.; Tetrahedron: Asymmetry 1996, 7, 2195.

16. Ward, D. E.; Rhee, C. K.; Tetrahedron Lett. 1991, 32, 7165. 